\title{
Acknowledgement to Reviewers of Administrative Sciences in 2018
}

\author{
Administrative Sciences Editorial Office \\ MDPI, St. Alban-Anlage 66, 4052 Basel, Switzerland \\ Published: 10 January 2019
}

Rigorous peer-review is the corner-stone of high-quality academic publishing. The editorial team greatly appreciates the reviewers who contributed their knowledge and expertise to the journal's editorial process over the past 12 months. In 2018, a total of 82 papers were published in the journal, with a median time to first decision of 21 days and a median time to publication of 56.5 days. The editors would like to express their sincere gratitude to the following reviewers for their cooperation and dedication in 2018:

Acker, Sandra
Adamides, Emmanuel
Adler, Nancy J.
Agnihotri, Arpita
Agosto, Vonzell
Agrizzi, Dila
Ahrne, Göran
Aldieri, Luigi
Aleksić, Ana
Alsaaty, Falih
Alston, Judy
Alziyadat, Naser
András, Gelei
Antonelli, Cristiano
Anysz, Hubert
Aparicio, Sebastian
Argenti, Paul A.
Arizkuren, Amaia
Backman, Charles
Badulescu, Alina
Bahrami, Shahab
Banaitis, Audrius
Baraibar-Diez, Elisa
Bariviera, Aurelio F.
Barrett, Gillian
Barthel, Erich
Baswan, Sudhir
Bendle, Neil
Berggren, Christian
Blasco López, Francis
Borge-Diez, David
Botti, Antonio
Bracci, Enrico
Braccini, Alessio Maria
Andiand

Acker, Sandra

Adler, Nancy J.

Agnihotri, Arpit

Agrizzi, Dila

Ahrne, Göran

Alston, Judy

Alziyadat, Naser

Antonelli, Cristiano

Anysz, Hubert

Aparicio, Sebastian

Argenti, Paul A.

Arizkuren, Amaia

Backman, Charles

Badulescu, Alina

Bahrami, Shahab

Banaitis, Audrius

Baraibar-Diez, Elisa

Bariviera, Aurelio F.

Barrett, Gillian

Barthel, Erich

Baswan, Sudhir

Bendle, Neil

Berggren, Christian

Blasco López, Francis

Borge-Diez, David

Botti, Antonio

Braccini, Alessio Maria
Brand, Maryse

Broerse, Jacqueline

Bromfield, Joseph

Buchmann, Robert Andrei

Buffa, Federica

Burtonshaw-Gunn, Simon

Burvill, Samantha

Butnaru, Gina

Carini, Cristian

Castro, Maria Guilhermina

Ceptureanu, Sebastian Ion

Cerchione, Roberto

Chapman, Elizabeth

Chapple, Wendy

Chaudhury, Mo

Chen, Bowei

Choi (Jason), Tsan-Ming

Choo, Pei Ling

Chow, Danny

Cojuharenco, Irina

Colbourne, Rick

Cooper, Katherine

Correia, PedroMiguel Alves Ribeiro

Covell, Christine

Cuadrado-Ballesteros, Beatriz

Dalmarco, Gustavo

Dang, Chongyu

Daniel, Emmanuel

D'Auria, Anna

David, Miriam Elizabeth

Del Río Rama, María De La Cruz

Demartini, Paola

Dembińska, Izabela

Desai, Sreedhari
Dimitriou, Dimitrios

Doi, Hirokazu

Dziadosz, Agnieszka

Eddy, Pamela

Erkut, Burak

Esmaeili, Behzad

EspinoRodriguez, TomasFrancisco

Ettl, Kerstin

Ferraris, Alberto

Fini, Riccardo

Fleacă, Elena

Fletcher, Luke

Franco, Mário

Gajpal, Yuvraj

Galán Zazo, José Ignacio

Galati, Francesco

Garcia-Sanchez, Isabel-María

Genc, Elif

Ghulam, Mustafa

Giuliana, Iannaccone

Glabadanidis, Paskalis

Glenna, Leland L.

Godinho Antunes, Marina

Górecka, Aleksandra

Górska-Warsewicz, Hanna

Gray, Garry

Grimaldi, Michele

Growe, Roslin

Grzybowska, Katarzyna

Habek, Patrycja

Hall, C. Michael

Han, Junghee

Harmoinen, Sari

Harris, Howard 


\author{
Havaei, Farinaz \\ Hayes, John Paul \\ Hechavarría, Diana M. \\ Herold, Nico \\ Herrero Lázaro, Marta \\ Herrmann, Frank \\ Hoggett, Paul \\ Hoppe, Magnus \\ Houghton, Luke \\ $\mathrm{Hu}$, Jinyu \\ Huxtable-Thomas, Louisa \\ Irving, Justin \\ Jarvis, Carol \\ Jaspers, Jelle David \\ Jha, Chandan \\ Jia, Fu \\ Jimenez, Paul \\ Jones, Stephen \\ Jong, Martine De \\ Junjan, Veronica \\ Kaimann, Daniel \\ Kantono, Kevin \\ Kattel, Rainer \\ Khajeheian, Datis \\ Kim, Bowon \\ Kim, Seong Dae \\ Klumpp, Matthias \\ Knorr, Helena \\ Kohtamäki, Vuokko \\ Korsell, Lars \\ Koutropoulos, Apostolos \\ Lai, Peddy Pi-Ying \\ Lashley, Mary \\ Laudal, Thomas \\ Leitão, João \\ Leixnering, Stephan \\ LeMay, Steve \\ Lenk, Peter \\ Li, Frank \\ Lim, Seunghoo \\ Lin, Joanna \\ Lioukas, Spyros \\ Lo, Wei-Shuo \\ López-Fernández, Macarena \\ López-López, Daniel \\ Lopez-Rojas, Edgar Alonso \\ MacDonald, Adriane \\ Machado, Carolina \\ Maditinos, Dimitrios I. \\ Manganelli, Benedetto \\ Manikas, Andrew S. \\ Mann, Hanuv \\ Maqbool, Rashid \\ Maritz, Alex
}

Marović, Ivan

Martínez, Jesus Barrena

Mather, Carey

Maytorena-Sanchez, Eunice

Mendes, Luis

Meneses, Raquel F. Ch.

Mi, Ruihua

Micera, Roberto

Michalska-Dudek, Izabela

Michnik, Jerzy

Miller, Karen-Lee

Miras Rodríguez, María Del Mar

Monciardini, David

Mondéjar, Juan Antonio

Mønster, Dan

Moorosi, Pontso

Morea, Donato

Morganti, Pierfrancesco

Munro, Kirsty

Murmura, Federica

Murray, John

Nagarajan, Magesh

Nazarko, Lukasz

Nesticò, Antonio

Nguyen, Pascal

Nikolov, Atanas Nik

Olague, Jose T.

Olson, Josephine E.

Opdyke, Aaron

Özkazanç-Pan, Banu

Palos-Sánchez, Pedro

Panda, Bibhudutta

Pantazatos, Andreas

Paola, Pierfrancesco De

Papathanassis, Alexis

Pardo-del-Val, Manuela

Patel, Vibhuti N.

Patelli, Edoardo

Pérez-López, Raúl

Philbin, Simon P.

Pizzi, Simone

Qazi, Abroon

Radu, Laura-Diana

Raeesi, Ramin

Ranabahu, Nadeera

Roberts, Chris

Romero-Martínez, Ángel

Roper, Stephen

Rorie, Melissa

Rosli, Ainurul

Roy, Raja

Ruban, Dmitry

Ruel, Huub J.M.

Ruiz-Real, José Luis
Ruwhiu, Diane

Ryder, Nicholas

Salas, Jesus M.

Salazar Hernández, Blanca

Salmerón, Esther

Samu, Flóra

Sanchez, Lidia

Sanmartin, Paul

Saura, Jose Ramon

Savage, Barbara

Schoepfer, Andrea

Scott, Heather Inez Ricks

Sentuti, Annalisa

Sergeeva, Natalya

Serpa, Sandro

Shakeshaft, Charol

Shyr, Oliver F.

Siemienska, Renata

Simoes, Pedro

Sireli, Yesim

Skaggs, Sheryl

Skouloudis, Antonis

Sobocińska, Magdalena

Sorgner, Alina

Sousa, Carlos M.P.

Sousa, Maria José

Stanley, Gordon

Starnawska, Marzena

Szafranko, Elżbieta

Szczepankiewicz, Elżbieta

Sziebig, Gabor

Taillieu, Tharsi

Taj, Saud

Takács, Károly

Tarquinio, Lara

Tartaglione, Andrea Moretta

Tavassoli, Nader

Taysum, Alison

Tello-Gamarra, Jorge

Thompson, Piers

Thyroff, Anastasia

Tiron-Tudor, Adriana

Toderi, Stefano

Topa, Gabriela

Trappenburg, Margo

Triguero, Angela

Tsai, Wen-Hsien

Tseng, Ming-Lang

Turskis, Zenonas

Urbaniec, Maria

Vande Walle, Gudrun

Vanderstraeten, Alex

Verhofstadt, Elsy

Vershinina, Natalia A. 
Vesci, Massimiliano

Vidwans, Mohini

Voet, Joris Van Der

Wach, Krzysztof

Walburg, Christian

Wang, Qing

Wang, Weisha
Watts, Susan

Welch, Mindy

Wink, Rüdiger

Wohlgezogen, Franz

Wróblewski, Łukasz

Xheneti, Mirela

Yan, Ruiliang
Yeh, Tsu-Ming

Yu, Manzhu

Yu, Yang

Zappatore, Marco

Zhang, Su

Zhao, Xianbo

Zibarras, Lara

(C) 2019 by the authors. Licensee MDPI, Basel, Switzerland. This article is an open access article distributed under the terms and conditions of the Creative Commons Attribution (CC BY) license (http://creativecommons.org/licenses/by/4.0/). 